\title{
Evaluation of CMV and KAP promoters for driving the expression of human CYP4F2 in transgenic mice
}

\author{
GUANGRUI LAI ${ }^{1}$, XIAOLIANG LIU ${ }^{1}$, JINGJING WU ${ }^{1}$, HONG LIU $^{2}$ and YANYAN ZHAO ${ }^{1,2}$ \\ ${ }^{1}$ Department of Clinical Genetics, Shengjing Hospital of China Medical University, Shenyang, Liaoning; \\ ${ }^{2}$ Department of Medical Genetics, China Medical University, Shenyang, Liaoning, P.R. China
}

Received July 5, 2011; Accepted August 12, 2011

DOI: $10.3892 / \mathrm{ijmm} .2011 .787$

\begin{abstract}
A transgenic mouse model in which cytochrome $\mathrm{P} 450$ $4 \mathrm{~F} 2$ ( $C Y P 4 F 2)$ was expressed in multiple organs was expected to clarify the role of 20-hydroxyeicosatetraenoic acid (20-HETE) in the regulation of blood pressure, compared with our previously established kidney androgen-regulated protein (KAP) promoter CYP4F 2 transgenic mouse model which predominantly showed renal overexpression of CYP4F2. A novel $C Y P 4 F 2$ transgenic mouse model driven by the cytomegalovirus (CMV) promoter was generated and identified by PCR and subsequent sequencing. Extensive study of CMV-CYP4F2 transgenic mice demonstrated that CYP4F2 was exclusively expressed in the liver, while 20-HETE levels in the urine, kidney and blood were not affected, and there was no resulting change in the systolic blood pressure. This was in contrast to KAP-CYP4F2 transgenic mice which exerted prohypertensive action of 20-HETE resulting from the renal overexpression of CYP4F2. In addition, CYP4F2 overwhelmed the endogenous renal $C y p 4 a$ family mRNA levels in the KAP-CYP4F2 but not in the CMV-CYP4F2 transgenic mice. These results support the idea that overexpression of renal CYP4F2, leading to high 20-HETE in the urine and blood, may account for the elevated blood pressure. The CMV promoter did not direct CYP4F2 expression into extensive tissues and organs in an attempt to clarify the action of 20-HETE.
\end{abstract}

\section{Introduction}

The human cytochrome P450 4F2 (CYP4F2) gene (MIM no. 604426) encodes an $\omega$-hydroxylase that catalyzes the

Correspondence to: Professor Yanyan Zhao, Department of Clinical Genetics, Shengjing Hospital of China Medical University, 36 San Hao Street, Shenyang, Liaoning 110004, P.R. China E-mail: yyzhao@sj-hospital.org; yyzhao@mail.cmu.edu.cn

Abbreviations: CYP4F2, cytochrome P450 4F2; KAP, kidney androgen-regulated protein; 20-HETE, 20-hydroxyeicosatetraenoic acid; CMV, cytomegalovirus; SBP, systolic blood pressure; TG, transgenic; WT, wild-type; LC-MS/MS, liquid chromatographytandem mass spectrometry

Key words: CYP4F2, 20-HETE, KAP, CMV metabolism of arachidonic acid to 20-hydroxyeicosatetraenoic acid (20-HETE) (1). Previous studies have demonstrated that 20-HETE has two contradictory effects in the development of hypertension $(2,3)$. One is prohypertension resulting from constriction of the small arteries $(4,5)$. The other is anti-hypertension caused by inhibition of sodium reabsorption $(6,7)$. We have previously elucidated the prohypertensive action of 20-HETE derived from CYP4F2 in the human population and in a transgenic mouse model $(8,9)$. We initially identified that a functional haplotype of $C Y P 4 F 2$ with increased transcriptional activity is associated with elevated urinary 20-HETE and hypertension in the Chinese population (8). Then, we successfully generated $C Y P 4 F 2$ transgenic mice of the $\mathrm{FVB} / \mathrm{N}$ strain with the kidney androgen-regulated protein (KAP) promoter (KAP-CYP4F2), and documented that the preferential renal overexpression of CYP4F2 enhances 20-HETE production and elevates systolic blood pressure (SBP) (9). Nevertheless, the following problems should be further addressed: i) whether or not the FVB/N genetic background is involved in the phenotype of the transgenic mice and ii) assessment of the phenotype of transgenic mice with strong CYP4F2 overexpression in multiple tissues and organs.

$\mathrm{FVB} / \mathrm{N}$ mice, the origin of the KAP-CYP4F2 transgenic mouse model, have higher renal 20-HETE production compared with BALB/c and C57BL/6 mice (10). The extent of 20-HETE production is possibly related to its prohypertension. Substantiating this possibility, renal overproduction of 20-HETE in mice $(9,11)$ and rats $(12-14)$ has been associated with the development of hypertension. Female mice, which have very low renal 20-HETE production, are more resistant to angiotensin II-induced hypertension (15). C57BL/6 males, which have lower renal 20-HETE production than the males of other strains, show reduced susceptibilities to the development of deoxycorticosterone acetate-salt hypertension (16). The BCF1 strain, a hybrid of BALB/c and C57BL/6 mice with reduced renal 20-HETE production compared to the FVB/N strain, was used to obtain a congenic $C Y P 4 F 2$ transgenic mouse model in which CYP4F2 expression was driven by the cytomegalovirus (CMV) promoter, which is widely used for directing the purposed gene expression into multiple tissues $(17,18)$. These CMV-CYP4F2 transgenic mice were expected to have higher 20-HETE levels in multiple tissues and organs, thus leading to higher SBP than KAP-CYP4F2 transgenic mice. 
In this study, CMV-CYP4F2 transgenic mice were compared to KAP-CYP4F2 transgenic mice in order to investigate the effect of 20-HETE on blood pressure. We also evaluated the impact of foreign $C Y P 4 F 2$ on endogenous Cyp4a10, Cyp4a12 and Cyp4a14 in mice.

\section{Materials and methods}

Generation of CMV-CYP4F2 transgenic mice. The novel CMV-CYP4F2 transgenic mouse model was generated according to previously described methods (9). In brief, CMV-CYP4F2/his plasmid was microinjected into BCF1 one-cell fertilized mouse embryos to generate two $C Y P 4 F 2$ transgenic founders. Mice were fed with standard mouse chow and water, bred in a 12:12 h light-dark cycle system. Transgenic mice, 14- to 20-weeks-old, were used for data collection, with age- and gender-matched wild-type mice as controls. All experiments conformed to the Guide for the Care and Use of Laboratory Animals published by the US National Institutes of Health (NIH Publication no. 85-23, revised in 1996). CYP4F2 cDNA was identified by PCR and confirmed by sequencing. PCR was performed using DNA isolated from tail biopsies with specific primers 5'-TGGACCTACGCCTTCTATGA-3' and 5'-CCACTTGTCACCAGCACTCA-3'.

Western blot analysis. Total protein was extracted from tissues of transgenic mice and the concentration was determined with the Bradford method. Denatured protein was separated by electrophoresis and transferred onto PVDF membranes (BioRad, USA) at $4^{\circ} \mathrm{C}$. Membranes were subsequently incubated with 1:1,000 anti-CYP4F2 antibody (Fitzgerald, Concord, MA, USA) or 1:1,000 anti- $\beta$-actin (Santa Cruz Biotechnology, Santa Cruz, CA, USA) as the primary antibodies, followed by alkaline phosphatase-conjugated $\operatorname{IgG}(1: 2,000)$ as the secondary antibody. The final detection reaction was performed with $\beta$-naphthyl acid phosphate and Fast Blue B salt (Sigma-Aldrich, St. Louis, MO).

20-HETE analysis by liquid chromatography-tandem mass spectrometry (LC-MS/MS). 20-HETE was measured by the API 3200 Q-trap LC-MS/MS system (Applied Biosystems, Foster City, CA) as previously reported (19). Generally, urine samples were incubated at $37^{\circ} \mathrm{C}$ for $2 \mathrm{~h}$ with $0.1 \mathrm{mg} / \mathrm{ml}$ $\beta$-glucuronidase (Sigma-Aldrich); $0.4 \mathrm{ml}$ blood samples were used without additional disposing; the kidney or liver samples were homogenated in methanol ( $0.1 \%$ formic acid), followed by addition of 2 ng 20-HETE-d6 (Cayman Chemical, Ann Arbor, Mich) to each sample as an internal standard. Lipids were extracted with ethyl acetate, dried under nitrogen, and resuspended in methanol. Samples were separated on a reversephase Symmetry C18 column $(3.5 \mu \mathrm{m}, 2.1 \times 150 \mathrm{~mm}$; Waters, Milford, MA) at a flow rate of $0.2 \mathrm{ml} / \mathrm{min}$ using solvent A (water, $0.1 \%$ formic acid) and solvent $\mathrm{B}$ (acetonitrile: methanol $6: 1,0.1 \%$ formic acid) $(0-2 \min 25 \% \mathrm{~B}, 2-10 \min 25-75 \% \mathrm{~B}$, $10-18 \min 75-95 \%$ B, $18-30 \min 95 \%$ B, 30-30.5 min 95-25\% $B$ and $30.5-40 \min 25 \%$ B). The effluent was ionized using negative ion electrospray and quantified by multiple reaction monitoring. The ion abundance of 20-HETE in the peaks vs. that of 20-HETE-d6 were determined and compared with standard curves generated over a range from 0.2 to $10 \mathrm{ng}$.
A

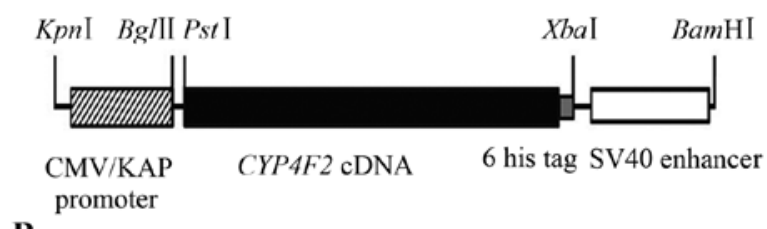

B

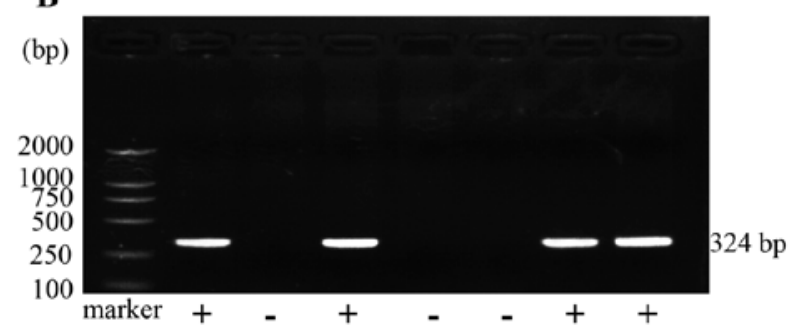

Figure 1. Schematic map of the linear CMV/KAP-CYP4F2/his and PCR analysis of $C Y P 4 F 2$ in transgenic mice. (A) The fragment for microinjection contained the CMV/KAP promoter, $C Y P 4 F 2$ cDNA, 6-his tag, and SV40 enhancer. (B) The agarose gel electrophoresis of PCR product.,$+ C Y P 4 F 2$ positive transgenic mice; -, $C Y P 4 F 2$ negative wild-type mice.

Blood pressure measurement. The mouse blood pressure was measured by the tail-cuff method with the IITC Life Science Model 1631 tail pulse detection system (IITC Life Science, Woodland Hills, CA, USA). Each mouse was warmed at $33^{\circ} \mathrm{C}$ in the heating container for $5 \mathrm{~min}$ before measurement. One measurement session involved 6 repetitions and at least three sessions were performed on each mouse to calculate the average.

Real-time PCR for mouse Cyp4a isoforms. Total RNA was isolated from kidney samples using TRIzol reagent (Invitrogen, Carlsbad, CA), and transcribed into cDNA with reverse transcription reagent kit (Promega, Madison, WI). Real-time PCR was performed on the ABI 7900 System (Applied Biosystems) in a $10 \mu \mathrm{l}$ SYBR-Green PCR reaction containing $1 \mathrm{X}$ SYBRGreen PCR master mix (Applied Biosystems), 5 ng cDNA, and $50 \mathrm{nM}$ forward and reverse primers synthesized specific to Cyp4a10, 5'-GACAAGGACCTACGTGCTGAGG-3' and 5'-CTCATAGCAAATTGTTTCCCA-3'; Cyp4a 12, 5'-GCA CAATCTCTTTTTTCTCCGTGTG-3' and 5'-GCAGGCACT GTTGGCCAA-3'; Cyp4a14, 5'-CCTACAAGGTACTTG GATGGT-3' and 5'-ATCATAAAGCAGGACTCGTATA-3'; and $\beta$-actin, 5'-TACCAACTGGGACGACATGG-3' and 5'-GGAGTCCATCACAATGCCTG-3'. The reaction condition was $95^{\circ} \mathrm{C}$ for $10 \mathrm{~min}$ followed by 40 cycles of $95^{\circ} \mathrm{C}$ for $15 \mathrm{sec}, 60^{\circ} \mathrm{C}$ for $1 \mathrm{~min}$, and $72^{\circ} \mathrm{C}$ for $30 \mathrm{sec}$. Dissociation curves were generated to ensure that a single and specific product was amplified. Cycle threshold values $(\mathrm{Ct})$ were analyzed by the SDS2.4 software (Applied Biosystems) and relative quantification of Cyp $4 a$ expression was determined using the comparative $\mathrm{Ct}$ method with the $\beta$-actin transcript as an internal control.

Statistical analysis. Data are expressed as means \pm SD. All data were analyzed by software SPSS17.0. We used the one-way analysis of variance or the Student's unpaired twotailed test for statistical analysis. Statistical significance was set at $\mathrm{P}<0.05$. 
A

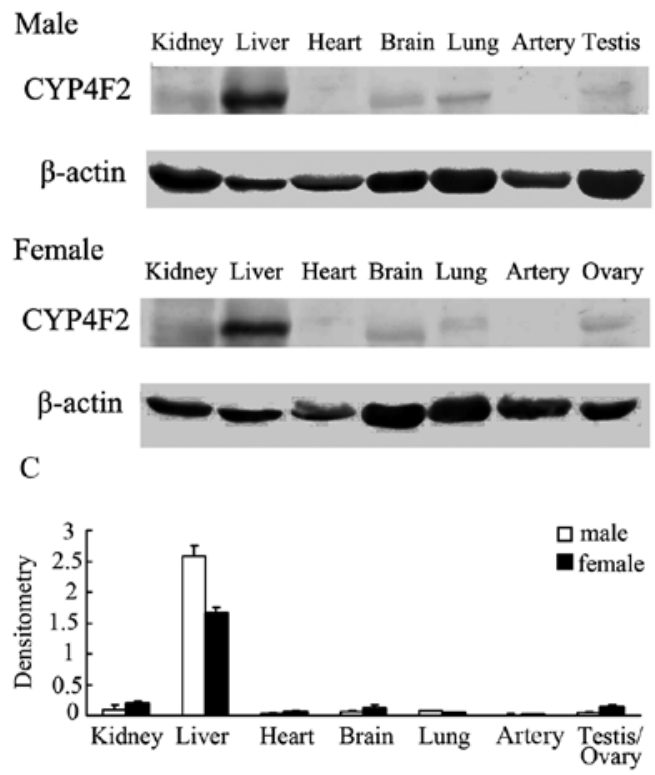

B

Male

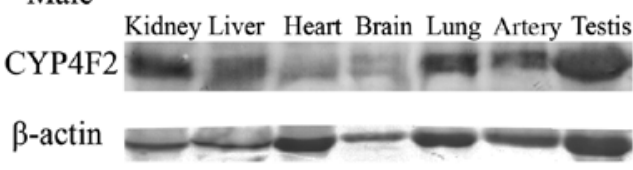

Female Kidney Liver Heart Brain Lung Artery Ovary

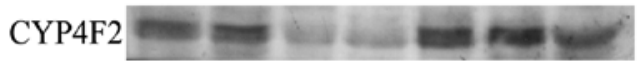

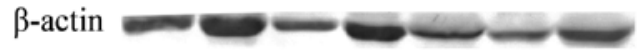

D

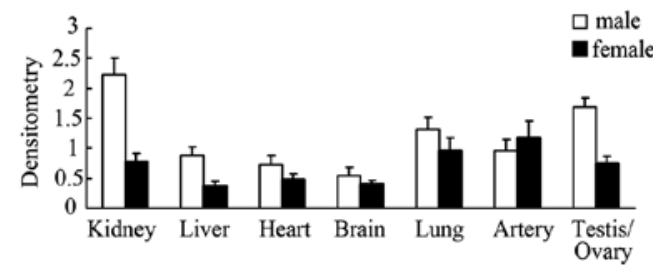

$\mathrm{E}$

Male

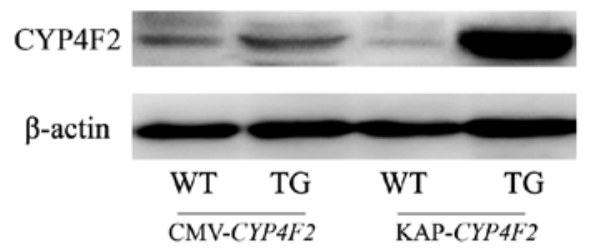

F

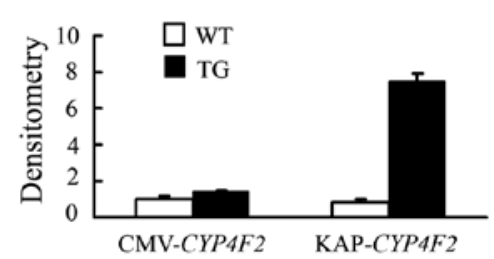

Female
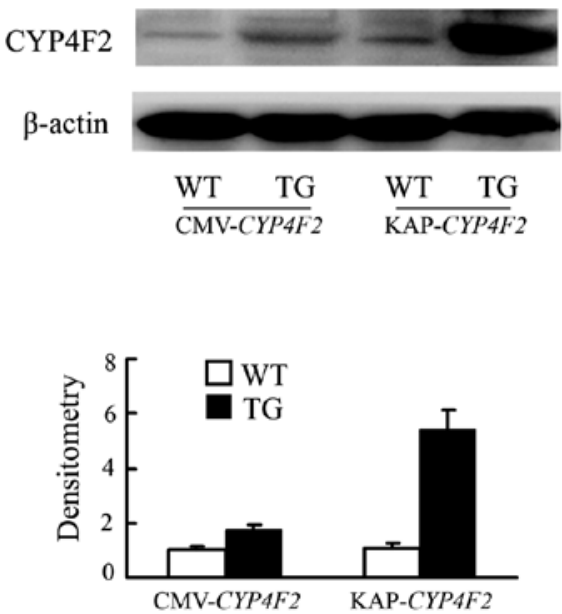

Figure 2. CYP4F2 expression by Western blot analysis. CYP4F2 was detected by an anti-CYP4F2 antibody, and $\beta$-actin was used as internal control. (A) CYP4F2 expression profile of male and female CMV-CYP4F2 transgenic mice. (B) CYP4F2 expression profile of male and female KAP-CYP4F2 transgenic mice. (C and D) Scanning densitometry of (A and B), respectively. (E) CYP4F2 expression in the kidney of the two types of transgenic (TG) mice and respective wild-type (WT) control. (F) Scanning densitometry of (E). All experiments were independently performed at least three times (n=3).

\section{Results}

Generation of $C M V-C Y P 4 F 2$ transgenic mice. In accordance with the previous strategy of generating the KAP-CYP4F2 transgenic mice (9), we currently produced the novel CMV-CYP4F2 transgenic mouse model (two founders) by the microinjection of the linearized CMV-CYP4F2/his DNA into the BCF1 strain. The difference of the construct plasmids between the two types of transgenic mice was a substitution of the KAP promoter by the CMV promoter (Fig. 1A). We routinely screened the CMV-CYP4F2 transgenic mice by PCR with primers specific for the CMV promoter and $C Y P 4 F 2$ to verify the transgene incorporation (Fig. 1B), and further confirmed the accuracy of $C Y P 4 F 2$ by sequencing. There was no apparent difference in the breeding and physiology between the CMV-CYP4F2 transgenic and wild-type mice; furthermore there was no difference in the data described below among the two lines of transgenic mice.

Analysis of CYP4F2 expression profile in transgenic mice. As illustrated in Fig. 2A and C, Western blot analysis showed that CYP4F2 expression was the highest in the liver tissue of CMV-CYP4F2 transgenic mice. The kidney and other tissues had subtle CYP4F2 expression. Furthermore, the expression profile of the $\mathrm{CMV}-C Y P 4 F 2$ transgenic mice was compared with that of the KAP-CYP4F2 transgenic mice. A prominent CYP4F2 expression in the kidney and sexual-hormone responsive tissues was detected in the KAP-CYP4F2 mice which was not observed in the CMV-CYP4F2 mice (Fig. 2B and D). We quantified the CYP4F2 levels in the kidneys of the two types of transgenic mice and confirmed that CMV-CYP4F2 


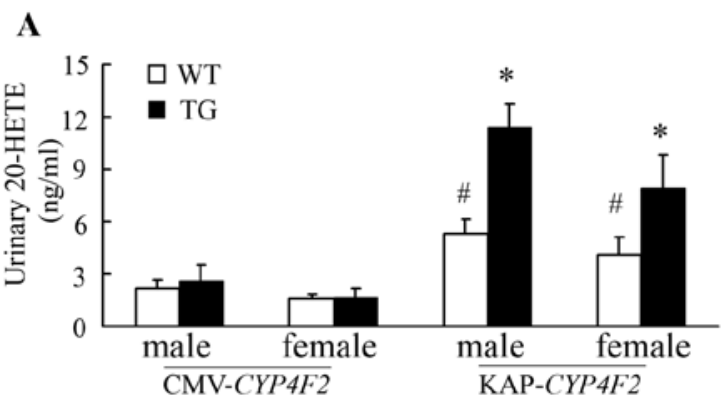

C

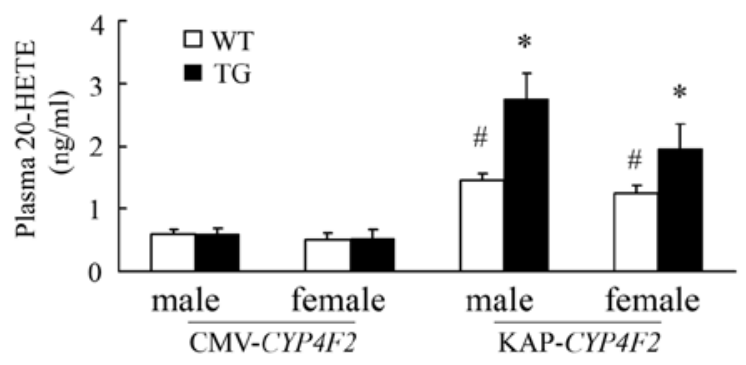

B

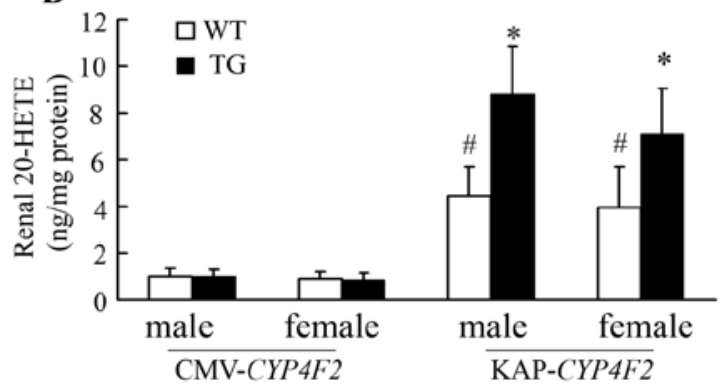

D

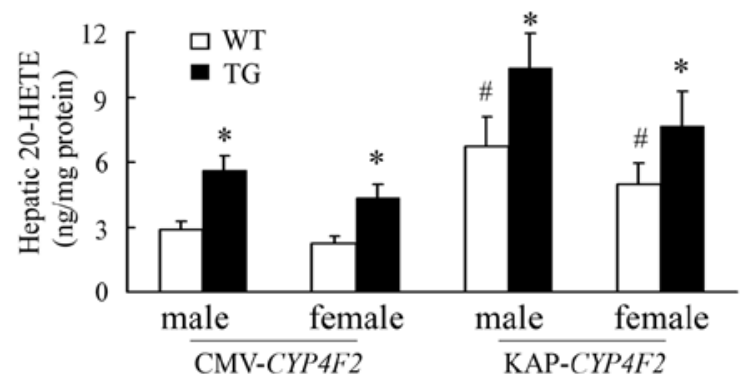

Figure 3. 20-HETE levels in (A) urine, (B) kidney, (C) plasma and (D) liver of CMV-CYP4F2 and KAP-CYP4F2 mice. TG, transgenic; WT, wild-type. "P<0.05 vs. KAP-CYP4F2 WT mice; ${ }^{*} \mathrm{P}<0.05$ vs. CMV-CYP4F2 WT mice $(\mathrm{n}=6)$.

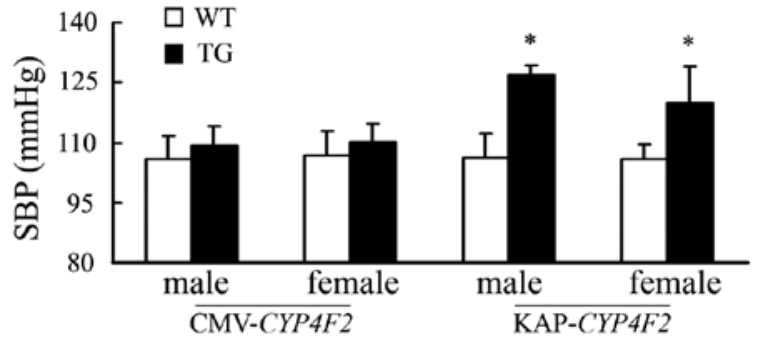

Figure 4. Systolic blood pressure (SBP) in the CMV-CYP4F2 and KAPCYP4F2 mice. TG, transgenic; WT, wild-type. " $\mathrm{P}<0.05$ vs. WT ( $\mathrm{n}=6)$.

mice had much lower expression levels of CYP4F2 than the KAP-CYP4F2 mice (Fig. 2E and F).

20-HETE level of the transgenic mice. 20-HETE is a bioactive molecule of CYP4F2. To determine whether the difference in the CYP4F2 expression between the CMV-CYP4F2 and the KAP-CYP4F2 mice may affect the levels of 20-HETE production, we measured the levels of 20-HETE in the urine, kidney, plasma and liver by LC-MS/MS analysis. As shown in Fig. 3, 20-HETE levels varied between wild-type BCF1 and FVB/N mice,with lower 20-HETE levels observed in the BCF1 than in $\mathrm{FVB} / \mathrm{N}$ strain. The CMV-CYP4F2 transgenic mice had nearly the same 20-HETE levels in the detected samples as the wild-type mice except in the liver (nearly 2-fold vs. control in both genders), and KAP-CYP4F2 transgenic mice had higher 20-HETE levels than wild-type mice, about 2.2-, 1.9-, 1.8- and 1.5 -fold in the urine, kidney, blood and liver for the male mice, respectively; and 2.0-, 1.8-, 1.6- and 1.5-fold for the female mice. The data demonstrate that $\mathrm{CYP} 4 \mathrm{~F} 2$ expression was in parallel with 20-HETE output in both CMV-CYP4F2 and KAP-CYP4F2 transgenic mice.
Blood pressure in transgenic mice. We previously reported that prominent CYP4F2 expression in the kidney of KAP-CYP4F2 transgenic mice leads to an increase of 20-HETE biosynthesis and systolic blood pressure. The systolic blood pressure values in the two types of transgenic mice studied herein are presented in Fig. 4. No differences in the systolic blood pressure were observed between CMV-CYP4F2 transgenic mice and their control mice in either gender. In contrast, KAP-CYP4F2 transgenic mice had higher blood pressure than their control mice in both genders $(21$ and $14 \mathrm{mmHg}$ higher in male and female respectively). These results indicate that the novel CMV-CYP4F2 transgenic mice did not have a hypertensive phenotype.

Renal endogenous Cyp 4 a isoforms in transgenic mice. To address whether the endogenous Cyp $4 a$ isoforms were involved in two types of transgenic mice, we measured the mRNA levels of Cyp4a10, Cyp4a12 and Cyp4a14 (Fig. 5A, B and C, respectively) in the kidney by real-time PCR. Comparison of CMV-CYP4F2 transgenic mice with wild-type mice showed no significant differences in the mRNA levels of three Cyp $4 a$ isoforms. In contrast, mRNA levels of three Cyp $4 a$ isoforms were lower in KAP-CYP4F2 transgenic mice than in the wild-type mice except for Cyp $4 a 12$ in females and Cyp $4 a 14$ in males, implying that foreign $C Y P 4 F 2$ could affect endogenous Cyp $4 a$ isoforms. In addition, BCF1 mice had much lower renal expression of almost all the Cyp $4 a$ isoforms than the $\mathrm{FVB} / \mathrm{N}$ mice in both genders.

\section{Discussion}

In this study, a novel $C Y P 4 F 2$ transgenic mouse model was generated under the control of the CMV promoter, showing no hypertensive phenotype, despite the increased CYP4F2 
A
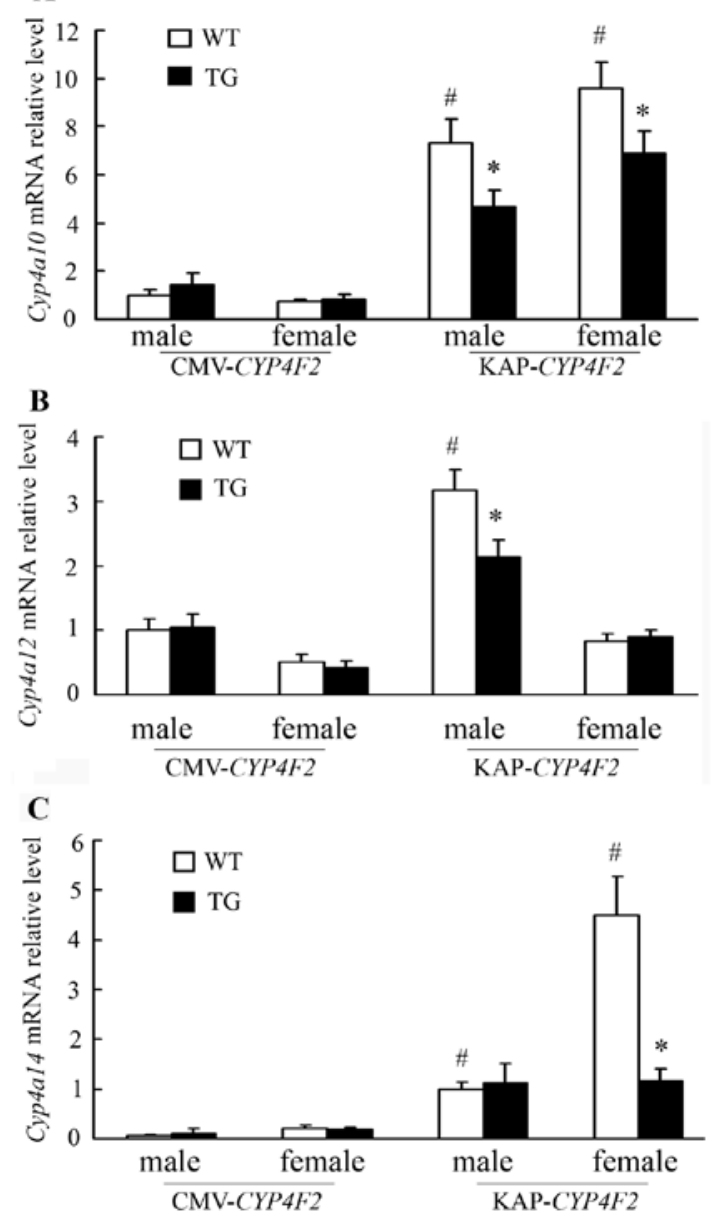

Figure 5. Relative expression of renal Cyp $4 a$ isoforms by real-time PCR. (A) Cyp4a10, (B) Cyp4a12 and (C) Cyp4a14 in CMV-CYP4F2 and KAPCYP4F2 mice. TG, transgenic; WT, wild-type, ${ }^{*} \mathrm{P}<0.05$ vs. KAP-CYP4F 2 WT mice; ${ }^{*} \mathrm{P}<0.05$ vs.CMV-CYP4F2 WT mice $(\mathrm{n}=6)$.

expression and 20-HETE production in the liver. These CMV-CYP4F2 transgenic mice were in striking contrast to KAP-CYP4F2 transgenic mice which exerted a prohypertensive action of 20-HETE resulting from renal overexpression of CYP4F2.

Native CYP4F2 is predominantly expressed in the kidney and liver, and has high $\omega$-hydroxylation activity to catalyze arachidonic acid to 20-HETE (1-3). 20-HETE promotes hypertension by vasoconstriction $(4,5)$ and attenuates hypertension by the inhibition of ion reabsorption $(6,7)$. The dual effect on regulation of blood pressure is spotlighted in the pathophysiology of hypertension. We previously generated a $C Y P 4 F 2$ transgenic mouse model under the control of a KAP promoter, and demonstrated that the renal overexpression of CYP4F2 contributes to increased 20-HETE production and elevated blood pressure in the KAP-CYP4F2 transgenic mice (9). In the current study, we attempted to use the CMV promoter for directing CYP4F2 expression into universal tissue of transgenic mice, but interestingly found a major expression in the liver where native CYP4F2 is primarily located. Compared to the KAP-CYP4F2 transgenic mice, the CMV-CYP4F2 transgenic mice had lower levels of CYP4F2 expression and 20-HETE production in the liver, and no change of 20-HETE in the urine, kidney and blood. Subsequently, we did not observe a hypertensive phenotype in the CMV-CYP4F2 transgenic mice. We postulated that 20-HETE located in the liver has a minimal function in the regulation of blood pressure due to the lack of the receptor or the effector downstream of 20-HETE. Nevertheless, we still inferred that renal 20-HETE exerts a dominant prohypertensive action from the contrary side of the CMV-CYP4F 2 model, which had normal 20-HETE in the urine and in the kidney and normal blood pressure compared to wild-type mice. From the data above, we conclude that the CYP4F2/20-HETE in the kidney rather than in the liver plays a critical role in the regulation of blood pressure. Since the CMV-CYP4F2 model has a subtle expression of CYP4F2 in the artery we could not evaluate action of 20-HETE derived from CYP4F2 on the vasculature.

It has been reported that the Cyp4a14-knockout mice show high levels of Cyp4a12 and 20-HETE in the kidney which results in hypertension (11), and mice with a dysfunctional Cyp 4 alo gene become salt-sensitive hypertensive even when fed with a normal salt diet (20), suggesting an interaction between the isoforms of 20-HETE synthesis involved in hypertension. To address the issue whether endogenous 20-HETE synthesis influenced the phenotype of the two types of transgenic mice, we assessed three isoforms in the mouse kidney, namely Cyp4a10, - $4 a 12$ and $-4 a 14$ by real-time PCR. The data demonstrated that the trangene $C Y P 4 F 2$ had no effect on the renal expression of three Cyp $4 a$ isoforms in CMV-CYP4F 2 mice, but interfered with Cyp $4 a 10$ expression in both genders, Cyp4a12 in male and Cyp4a14 in the female KAP-CYP4F2 mice. Among the three isoforms, Cyp $4 a 10$ is highly expressed in the kidneys of both genders, Cyp4a12 is predominantly a male- and Cyp $4 a 14$ a female-specific isoform (10). Therefore, we speculated that the high renal expression of CYP4F2 overwhelmed their dominant expression in the kidney of the KAP-CYP4F2 mice. In addition, we also found that the $\mathrm{BCF} 1$ strain, the origin of $\mathrm{CMV}-C Y P 4 F 2$ transgenic mice, had much lower renal expression of almost all the Cyp $4 a$ isoforms than the $\mathrm{FVB} / \mathrm{N}$ strain which is the origin of KAP-CYP4F2 transgenic mice, coinciding with the lower urinary and renal 20-HETE in BCF1 than in the FVB/N mice.

The CMV promoter is a powerful and promiscuous promoter widely used for driving the purposed gene expression (21). Some studies on foreign genes under the control of the CMV promoter described universal tissue expression in transgenic mice $(17,18)$, and others reported that gene expression with the CMV promoter seems to be variable and tissue-dependent in transgenic mice $(22,23)$. In our CMV-CYP4F2 model, CYP4F2 expression was found to occur predominantly in the liver, although other tissues had subtle expression including the kidney. Extensive study of CMV-CYP4F2 mice revealed no change in 20-HETE levels of the urine and kidney and in the blood pressure. Because these findings were consistent in two transgenic lines, this pattern of expression is unlikely to be attributable to the site of transgene insertion. Similar findings that transgene IGF-I expression was restricted exclusively to the testis and OX40-Ig and GK-Ig expression was relatively specific to the pancreas in CMV promoter-directed transgenic models have been reported by Dyck et al (22) and Zhan et al (23), respectively. Mohammed et al (24) have suggested that the CMV promoter 
sequence itself is not sufficient to determine tissue or species specificity and may be involved in differential methylation. Therefore, more studies are needed to explain why CMV controls the liver-specific expression of CYP4F2 in the CMV-CYP4F2 model. As to the KAP promoter, we previously showed robust renal and moderate extra-renal expression in the KAP-CYP4F2 model (9). Such pattern of transgene expression occurred in KAP-hATG transgenic mice with high expression of angiotensinogen in the kidney and epididymis (25), in KAP2-iCre transgenic mice with high levels of improved Cre in the kidney and low levels in the brain and liver (26), and in the KAP- $h R E N$ transgenic mice with predominant expression of renin in the kidney and variable in other tissues (27). Taken together, the marker genes controlled by the KAP promoter were all highly expressed in the kidneys of all the transgenic mice, including our KAP-CYP4F2 transgenic mice. Thus, the KAP promoter is a selective kidney-specific promoter and is a wiser option for the CYP4F2-targeted kidney.

In summary, our results reinforce the idea that the overexpression of renal CYP4F2, leading to high 20-HETE, may account for the elevated blood pressure. The foreign CYP4F2, if highly expressed in the kidney, can overwhelm the endogenous renal Cyp4a gene in the transgenic mice. In addition, KAP but not the CMV promoter can efficiently drive the expression of kidney-specific genes.

\section{Acknowledgements}

This study was supported by the ' 973 ' Project of China (Grant no. 2009CB526401) and the National Natural Science Foundation of China (Grant nos. 81070206 and 30800463 ).

\section{References}

1. Lasker JM, Chen WB, Wolf I, Bloswick BP, Wilson PD and Powell PK: Formation of 20-hydroxyeicosatetraenoic acid, a vasoactive and natriuretic eicosanoid, in human kidney. Role of CYP4F2 and CYP4A11. J Biol Chem 275: 4118-4126, 2000.

2. Williams JM, Murphy S, Burke M and Roman RJ: 20-Hydroxyeicosatetraeonic acid: a new target for the treatment of hypertension. J Cardiovasc Pharmacol 56: 336-344, 2010.

3. Zordoky BN and El-Kadi AO: Effect of cytochrome P450 polymorphism on arachidonic acid metabolism and their impact on cardiovascular diseases. Pharmacol Ther 125 : 446-463, 2010.

4. Inoue K, Sodhi K, Puri N, et al: Endothelial specific CYP4A2 overexpression leads to renal injury and hypertension via increased production of 20-HETE. Am J Physiol Renal Physiol 297: 875-884, 2009.

5. Sodhi K, Wu CC, Cheng J, et al: CYP4A2-induced hypertension is 20-hydroxyeicosatetraenoic acid- and angiotensin II-dependent. Hypertension 56: 871-878, 2010.

6. Quigley R, Baum M, Reddy KM, Griener JC and Falck JR: Effects of 20-HETE and 19(S)-HETE on rabbit proximal straight tubule volume transport. Am J Physiol 278: 949-953, 2000.

7. Zhou Y, Huang H, Chang HH, Du J, Wu JF, Wang CY and Wang MH: Induction of renal 20-hydroxyeicosatetraenoic acid by clofibrate attenuates high-fat diet-induced hypertension in rats. J Pharmacol Exp Ther 317: 11-18, 2006.

8. Liu H,Zhao Y, Nie D, et al: Association of a functional cytochrome P4504F2 haplotype with urinary 20-HETE and hypertension. J Am Soc Nephrol 19: 714-721, 2008.
9. Liu X, ZhaoY, Wang L, et al: Overexpression of cytochrome P450 $4 \mathrm{~F} 2$ in mice increases 20 -hydroxyeicosatetraenoic acid production and arterial blood pressure. Kidney Int 75: 1288-1296, 2009.

10. Dominik NM, Cosima S, Eduardo B, et al: Mouse Cyp4a isoforms: enzymatic properties, gender- and strain-specific expression, and role in renal 20-hydroxyeicosatetraenoic acid formation. Biochem J 403: 109-118, 2007.

11. Fidelis P, Wilson L, Thomas K, Villalobos M and Oyekan AO: Renal function and vasomotor activity in mice lacking the Cyp4a14 gene. Exp Biol Med 235: 1365-1374, 2010.

12. Zhang F, Chen CL, Qian JQ, Yan JT, Cianflone K, Xiao X and Wang DW: Long-term modifications of blood pressure in normotensive and spontaneously hypertensive rats by gene delivery of rAAV-mediated cytochrome P450 arachidonic acid hydroxylase. Cell Res 15: 717-724, 2005.

13. Gao Z, Koba S, Sinoway L and Li J: 20-HETE increases renal sympathetic nerve activity via activation of chemically and mechanically sensitive muscle afferents. J Physiol 586: 2581-2591, 2008.

14. Su P, Kaushal KM and Kroetz DL: Inhibition of renal arachidonic acid omega-hydroxylase activity with ABT reduces blood pressure in the SHR. Am J Physiol 275: 426-438, 1998.

15. Xue B, Pamidimukkala $J$ and Hay M: Sex differences in the development of angiotensin II-induced hypertension in conscious mice. Am J Physiol Heart Circ Physiol 288: 2177-2184, 2005.

16. Hartner A, Cordasic N, Klanke B, Veelken R and Hilgers KF: Strain differences in the development of hypertension and glomerular lesions induced by deoxycorticosterone acetate salt in mice. Nephrol Dial Transplant 18: 1999-2004, 2003.

17. Furth PA, Hennighausen L, Bakern C, Beatty B and Woychick R: The variability in activity of the universally expressed human cytomegalovirus immediate early gene 1 enhancer/promoter in transgenic mice. Nucleic Acids Res 19: 6205-6208, 1991.

18. Schmidt EV, Christoph G, Zeller R and Leder P: The cytomegalovirus enhancer: a pan-active control element in transgenic mice. Mol Cell Biol 10: 4406-4411, 1990.

19. Satoshi I, Victor G, Mohamad N, et al: L-4F differentially alters plasma levels of oxidized fatty acids resulting in more antiinflammatory HDL in mice. Drug Metab Lett 4: 139-148, 2010.

20. Kiyoshi N, Vijaykumar RH and Yuan W: Salt-sensitive hypertension is associated with dysfunctional Cyp $4 a 10$ gene and kidney epithelial sodium channel. J Clin Invest 116: 1696-1702, 2006.

21. Paulina W, Egbert B and Jens K: CMV promoter is inadequate for expression of mutant human RyR2 in transgenic rabbits. $\mathrm{J}$ Pharmacol Toxicol Methods 63: 180-185, 2011.

22. Dyck MK, Ouellet M, Gagn M, Petitclerc D, Sirard MA and Pothier F: Testes-specific transgene expression in insulin-like growth factor-I transgenic mice. Mol Reprod Dev 54: 32-42, 1999.

23. Zhan Y, Brady JL, Johnston AM and Lew AM: Predominant transgene expression in exocrine pancreas directed by the CMV promoter. DNA Cell Biol 19: 639-645, 2000.

24. Mohammed AD, Guisheng Z, Joseph EK and Dexi L: Evaluation of viral and mammalian promoters for driving transgene expression in mouse liver. Biochem Biophys Res Commun 339: 673-678, 2006.

25. Ding Y, Davisson RL, Hardy DO, Zhu LJ, Merrill DC, Catterall JF and Sigmund CD: The kidney androgen-regulated protein promoter confers renal proximal tubule cell-specific and highly androgen-responsive expression on the human angiotensinogen gene in transgenic mice. J Biol Chem 272: 28142-28148, 1997.

26. Li H, Zhou X, Davis DR, Xu D and Sigmund CD: An androgeninducible proximal tubule-specific Cre recombinase transgenic model. Am J Physiol Renal Physiol 294: 1481-1486, 2008.

27. Lavoie JL, Lake-Bruse KD and Sigmund CD: Increased blood pressure in transgenic mice expressing both human renin and angiotensinogen in the renal proximal tubule. Am J Physiol Renal Physiol 286: 965-971, 2004. 